\title{
IMPLICATIONS OF VISCOSITY AND STRAIN-GRADIENT EFFECTS FOR THE KINETICS OF PROPAGATING PHASE BOUNDARIES IN SOLIDS*
}

\author{
ROHAN ABEYARATNE $\dagger$ AND JAMES K. KNOWLES $\ddagger$
}

\begin{abstract}
This paper is concerned with the propagation of phase boundaries in elastic bars. It is known that the Riemann problem for an elastic bar capable of undergoing isothermal phase transitions need not have a unique solution, even in the presence of the requirement that the entropy of any particle cannot decrease upon crossing a phase boundary. For a special class of elastic materials, the authors have shown elsewhere that if all phase boundaries move subsonically with respect to both phases, this lack of uniqueness can be resolved by imposing a nucleation criterion and a kinetic relation for the relevant phase transition. Others have singled out acceptable solutions on the basis of a theory that adds effects due to viscosity and second strain gradient to the elastic part of the stress. It is shown that, for phase boundaries that propagate subsonically, this approach is equivalent to the imposition of a particular kinetic relation at the interface between the phases.
\end{abstract}

Key words. phase transitions, kinetics, Riemann problem

AMS(MOS) subject classifications. 73, 35

1. Introduction. In a recent paper [1], we considered longitudinal motions of an elastic bar according to a one-dimensional continuum model that permits the material of the bar to undergo phase transitions. Although inertial effects were taken into account in [1], motions were assumed to take place isothermally. When a motion of the bar involves a propagating strain discontinuity, the second law of thermodynamics imposes an entropy admissibility requirement: at each strain jump, the product of the velocity $\dot{s}$ of the discontinuity and an associated driving traction $f$ must be nonnegative: $f \dot{s} \geqq 0$.

If the material of the bar is such that stress is a monotonically increasing, strictly convex, or strictly concave function of strain, then phase transformations cannot occur, and all propagating strain discontinuities are shock waves. For a bar made of such a material, the Cauchy problem for the associated field equations and jump conditions has at most one solution that fulfills the entropy admissibility requirement.

On the other hand, for the class of materials considered in [1], the stress-strain relation is such that stress at first increases with increasing strain, then decreases and finally increases again. A constitutive law of this kind affords a continuum model for phase transitions in the present setting. A propagating strain discontinuity may now be either a shock wave or a phase boundary, according to whether the particles separated by the discontinuity are of the same phase or of distinct phases. In this setting, the Cauchy problem need no longer have a unique solution, even with the entropy admissibility requirement in force. For the Riemann problem, it was shown in [1] that this lack of uniqueness may be viewed as arising from the need to specify two additional pieces of constitutive information pertaining to phase boundaries: a nucleation criterion for the initiation of a phase transition and a kinetic relation that controls the rate at which the phase transition proceeds. The importance of nucleation and kinetics has

* Received by the editors June 25, 1990; accepted for publication (in revised form) October 22, 1990. This research was supported by U.S. Office of Naval Research contract N00014-87-K-0117.

†Department of Mechanical Engineering, Massachusetts Institute of Technology, Cambridge, Massachusetts 02139.

$\ddagger$ Division of Engineering and Applied Science, California Institute of Technology, Pasadena, California 91125. 
long been recognized in the materials science literature concerning phase transformations [2].

A similar need had been identified in an earlier study of quasi-static processes involving phase transformations in elastic bars [3]. The model for such processes given in [3] incorporates a nucleation criterion and a kinetic relation. It leads to a determinate quasi-static theory and yields results that are in qualitative agreement with experimental observations for bars made of "shape-memory" materials [4]. The form of the kinetic relation employed in [3] and [1] and to be used here is one in which the driving traction is a materially determined function of the velocity of the moving phase boundary: $f=\varphi(\dot{s})$. The nucleation criterion involves the specification of a critical level of driving traction that signals the onset of a phase transformation. The kinetic relation is a constitutive assertion that, for a given $\varphi$, is appropriate to a particular material. On the other hand, the entropy inequality $f \dot{s} \geqq 0$ at strain discontinuities follows from a fundamental physical principle and is therefore applicable to all materials.

For a special material whose rising-falling-rising stress-strain curve is piecewise linear, we showed in [1] that, for the Riemann problem, the extent of lack of uniqueness of solution remaining after imposition of the entropy admissibility requirement was precisely that needed to accommodate the nucleation criterion and the kinetic relation at phase boundaries, at least for kinetic relations of the kind introduced in [3] and in circumstances where the phase boundary propagates subsonically with respect to both phases. Kinetic relations may not be prescribed at shock waves. Phase boundaries that move supersonically with respect to the material phase with lower sound speed were also discussed in [1]; it was found that no kinetic relation may be prescribed at such a phase boundary in the Riemann problem.

A different approach to the issue of identifying meaningful solutions to a quasilinear system of dynamical field equations and jump conditions in the presence of propagating phase boundaries has been explored by Shearer [5]-[7], Slemrod [8]-[10], Truskinovsky [11]-[13], and others. Although these authors are often concerned with the van der Waals fluid, they note that their method can be applied to elastic bars as well. In this approach, one first augments the conventional constitutive law for an elastic material capable of undergoing isothermal phase transitions in such a way that the stress $\sigma$ depends not only on the strain $\gamma$, but also on the strain rate $\gamma_{t}$ and the second spatial strain gradient $\gamma_{x x}$. The dependence of $\sigma$ on $\gamma$ is nonlinear, but $\gamma_{t}$ and $\gamma_{x x}$ enter linearly and are associated with a viscosity $\nu$ and a strain-gradient coefficient $\lambda$, respectively. Next, one establishes a criterion to identify those moving phase boundaries within the elastic theory that are preferred on the basis of this augmentation: the criterion asserts that such a phase boundary is preferred if and only if the strains $\bar{\gamma}$ and $\stackrel{+}{\gamma}$ on either side of the discontinuity can be smoothly connected by a traveling wave constructed within the augmented theory.

The present paper is concerned with the relationship between the approach based on such an augmented model and the direct approach described in [1]. We show that the selection criterion based on this augmented model is equivalent to the prescription of a kinetic relation $f=\varphi(\dot{s})$ with a particular choice of $\varphi$.

In the next section, we formulate the elastic theory and its augmentation as described above. In $\S 3$, we review the local properties of strain discontinuities according to the elastic theory; as in [1], we impose a kinetic relation at phase boundaries. We consider the augmented theory in $\S \S 4-6$, where we study traveling waves when the strain dependence of the stress is trilinear. We show in $\S 5$ that strains $\bar{\gamma}$ and $\stackrel{+}{\gamma}$ can be connected by a subsonic traveling wave in the augmented theory if and only if they 
are related by a certain restriction. In contrast, no such restriction applies to strains that are connectable by a supersonic traveling wave (see $\S 6$ ). In $\S 7$, we delineate the class of phase boundaries within the elastic theory that are preferred by the criterion described above. In the subsonic case, we find that the preferred phase boundaries are those that conform to a particular kinetic relation that arises directly from the restriction mentioned above. This kinetic relation is of precisely the kind introduced in the quasi-static setting in [3] and studied in the dynamic context in [1]. For supersonic phase boundaries, no kinetic relation emerges from the augmented theory. The distinction betwen subsonic and supersonic phase boundaries observed here is entirely consistent with the findings reported in [1] and described briefly above.

2. Basic equations. Suppose that a bar of unit cross-sectional area occupies the interval $(-\infty, \infty)$ of the $x$-axis in a reference configuration. During a motion, the particle at $x$ in the reference configuration is carried to $x+u(x, t)$ at time $t$, where $u(x, t)$ is the displacement. We assume that $u$ is at least continuous with piecewise continuous first and second derivatives throughout the regions of space-time to be considered; more stringent requirements will often be needed. The strain $\gamma(x, t)$ and particle velocity $v(x, t)$ are defined by

$$
\gamma=u_{x}, \quad v=u_{t}
$$

wherever the derivatives exist. Balance of momentum and compatibility of $\gamma, v$ require that

$$
\begin{gathered}
\sigma_{x}=\rho v_{t}, \\
v_{x}=\gamma_{t},
\end{gathered}
$$

at points where $\sigma, v$, and $\gamma$ are smooth; here, $\sigma(x, t)$ is the stress at $(x, t)$, and $\rho$ is the (constant) mass density in the reference configuration. If either $\gamma$ or $v$ is discontinuous across a curve $x=s(t)$ in the $x, t$-plane, balance of momentum and the smoothness properties of $u$ yield the following jump conditions:

$$
\begin{gathered}
\llbracket \sigma \rrbracket=-\rho \dot{s} \llbracket v \rrbracket, \\
\llbracket v \rrbracket=-\dot{s} \llbracket \gamma \rrbracket,
\end{gathered}
$$

where $\dot{s}(t)$ is the velocity of the moving strain discontinuity, and for any $g(x, t)$ we have written $\llbracket g \rrbracket \equiv g(s(t)+, t)-g(s(t)-, t)$.

For the elastic bar, we take

$$
\sigma=\hat{\sigma}(\gamma)
$$

here $\hat{\sigma}(\gamma)$ is the stress response function whose graph is shown in Fig. 1. The strain $\gamma$ is restricted to the range $(-1,+\infty)$ to assure that the mapping $x \rightarrow x+u(x, t)$ is invertible for each $t$. We say that a particle of the bar labeled by $x$ is in phase 1, 2, or 3 at time $t$ according to whether the strain $\gamma(x, t)$ is in $\left(-1, \gamma_{M}\right],\left(\gamma_{M}, \gamma_{m}\right)$, or $\left[\gamma_{m}, \infty\right)$, respectively. The constants $\mu_{1}$ and $\mu_{3}<\mu_{1}$ are the elastic moduli, and $c_{1}=\left(\mu_{1} / \rho\right)^{1 / 2}$ and $c_{3}=\left(\mu_{3} / \rho\right)^{1 / 2}<c_{1}$ are the corresponding sound wavespeeds, in phases 1 and 3 . The special stress $\sigma_{0}$ shown in Fig. 1 is such that the two shaded areas are equal; it is called the Maxwell stress. For the trilinear material, $\sigma_{0}=\left(\sigma_{M} \sigma_{m}\right)^{1 / 2}$, where $\sigma_{M}$ and $\sigma_{m}$ are the respective stresses at the local maximum and minimum. On the declining branch of the stress-strain curve, $\hat{\sigma}(\gamma)=-\mu_{2} \gamma+\sigma_{2}$. We set $c_{2}=\left(\mu_{2} / \rho\right)^{1 / 2}$; note that $c_{2}$ is not a wavespeed. Continuity of $\hat{\sigma}(\gamma)$ at the maximum and the minimum requires that

$$
\sigma_{2} / \rho=\left(c_{1}^{2}+c_{2}^{2}\right) \gamma_{M}=\left(c_{3}^{2}+c_{2}^{2}\right) \gamma_{m}
$$




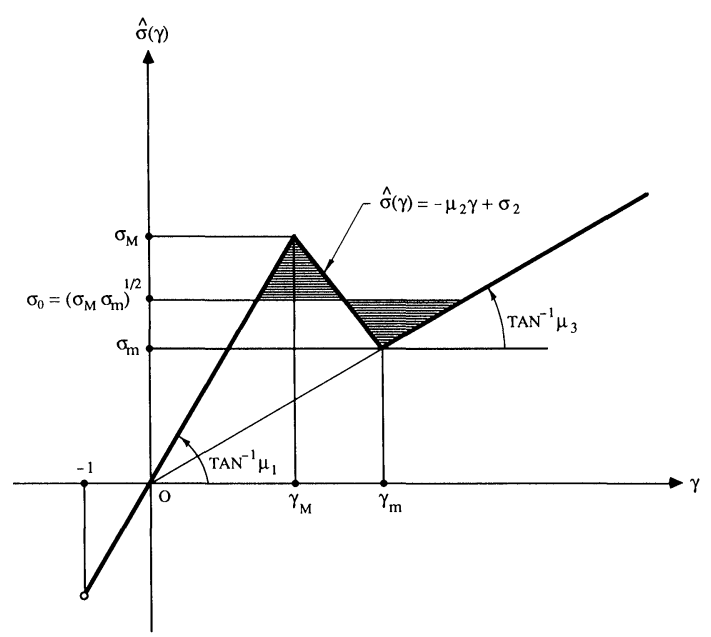

FIG. 1. Stress-strain curve for the trilinear material.

In the augmented theory, the constitutive statement (2.6) is replaced by

$$
\sigma=\hat{\sigma}(\gamma)+\rho \nu \gamma_{t}-\rho \lambda \gamma_{x x},
$$

where $\hat{\sigma}(\gamma)$ is again the trilinear function of Fig. 1 , and $\nu \geqq 0$ and $\lambda \geqq 0$ are the viscosity and the strain-gradient coefficient, respectively. The dimensionless parameter $\omega$ defined by

$$
\omega=2 \lambda^{1 / 2} / \nu
$$

will be useful in what follows.

When considering the material governed by (2.8), we will strengthen the smoothness requirements: $u$ will be twice continuously differentiable with piecewise continuous third and fourth derivatives in all parts of space-time to be considered. Of the jump conditions $(2.4),(2.5)$, the latter is then trivially satisfied, and the former reduces to the requirement that $\sigma$ be continuous; by (2.8), this requires that $\gamma_{x x}$, and therefore $u_{x x x}$, be continuous.

The augmented theory used here is analogous to a special case of a theory proposed by Korteweg [14] to account for the effect of capillarity in a viscous fluid (see [8]). In the latter theory, $\gamma$ in (2.8) is identified with specific volume, $-\sigma$ with pressure, $\rho$ with density, $\nu$ with viscosity, and $\lambda$ with capillarity. Thermo-mechanical difficulties raised by Korteweg's theory have been discussed in [15].

3. Local theory of phase boundaries in elastic bars. We now consider the elastic case (2.1)-(2.6), together with the appropriate smoothness assumptions. Although many of the detailed results to follow apply only to the trilinear material, the more general discussion in the first part of the present section, as well as certain subsequent results, are valid for any rising-falling-rising stress-strain curve.

Suppose that, during a motion, the portion of the bar occupying the interval $\left[x_{1}, x_{2}\right]$ in the reference configuration bears fields $\gamma, v$, and $\sigma$ that are smooth except for a single moving strain jump at $x=s(t)$. Let

$$
W(\gamma)=\int_{0}^{\gamma} \hat{\sigma}\left(\gamma^{\prime}\right) d \gamma^{\prime}, \quad \gamma>-1
$$


be the stored energy per unit volume of the bar, and consider the total mechanical energy

$$
E(t)=\int_{x_{1}}^{x_{2}}\left[W(\gamma)+\frac{1}{2} \rho v^{2}\right] d x
$$

associated with the piece of the bar under consideration at time $t$. A direct calculation using (3.1), (2.2)-(2.5) shows that

$$
\sigma\left(x_{2}, t\right) v\left(x_{2}, t\right)-\sigma\left(x_{1}, t\right) v\left(x_{1}, t\right)-\dot{E}(t)=f(t) \dot{s}(t),
$$

where the driving traction $f$ acting on the strain discontinuity is defined by

$$
f=\hat{f}(\bar{\gamma}, \stackrel{+}{\gamma}) \equiv \int_{\bar{\gamma}}^{+\dot{\gamma}} \hat{\sigma}(\gamma) d \gamma-\frac{1}{2}(\stackrel{+}{\sigma}+\bar{\sigma})(\stackrel{+}{\gamma}-\bar{\gamma}) .
$$

Here $\stackrel{ \pm}{\gamma}=\stackrel{ \pm}{\gamma}(t)=\gamma(s(t) \pm, t)$ and $\stackrel{ \pm}{\sigma}=\stackrel{ \pm}{\sigma}(t)=\sigma(s(t) \pm, t)$ are the strains and stresses, respectively, on the positive and negative sides of the strain discontinuity. The left side of (3.3) represents the excess of the rate of work of the external forces over the rate of increase of mechanical energy. Although this excess vanishes for smooth strain fields $(f=0)$ or for stationary discontinuities $(\dot{s}=0)$, this is not the case in general. If the material is viewed as thermoelastic, and if we make the assumption-however unrealistic - that the motions considered take place isothermally, then as shown in [16], the Clausius-Duhem inequality requires that the instantaneous rate of mechanical dissipation $f(t) \dot{s}(t)$ be nonnegative:

$$
f(t) \dot{s}(t) \geqq 0
$$

for all time. The entropy admissibility condition (3.5) must hold at all strain discontinuities; under isothermal conditions, it is equivalent to the assertion that the entropy of a particle cannot decrease as the particle crosses a discontinuity in strain.

At the moving discontinuity $x=s(t)$, the jump conditions (2.4), (2.5) imply that

$$
\begin{aligned}
& \rho \dot{s}^{2}=\frac{\hat{\sigma}(\stackrel{+}{\gamma})-\hat{\sigma}(\bar{\gamma})}{\stackrel{+}{\gamma}-\bar{\gamma}}, \\
& {[\hat{\sigma}(\stackrel{+}{\gamma})-\hat{\sigma}(\bar{\gamma})](\stackrel{+}{\gamma}-\bar{\gamma})=\rho(\stackrel{+}{v}-\bar{v})^{2} .}
\end{aligned}
$$

The right side of (3.6) is thus necessarily nonnegative for any pair of strains $\bar{\gamma}, \stackrel{+}{\gamma}$ that can occur at a strain jump.

Conversely, if $\bar{\gamma}, \stackrel{+}{\gamma}$ are numbers in $(-1, \infty)$ such that the right side of (3.6) is nonnegative, then it is possible to find numbers $\bar{v}, \stackrel{+}{v}$, and $\dot{s}$ such that the jump conditions (2.4), (2.5) are satisfied.

Because we are concerned here with phase boundaries rather than shock waves, we assume that $\bar{\gamma}$ and $\stackrel{+}{\gamma}$ belong to different phases. Since we will not consider cases in which either $\bar{\gamma}$ or $\stackrel{+}{\gamma}$ is in phase 2, i.e., the "unstable phase," we may as well take $\bar{\gamma}$ to be in phase 1 , and ${ }^{+}$in phase 3 . When specialized to this case and to the trilinear material of Fig. 1, (3.6) becomes

$$
\dot{s}^{2}=\frac{c_{3}^{2+} \gamma-c_{1}^{2} \bar{\gamma}}{\stackrel{+}{\gamma}-\bar{\gamma}}
$$

In the $\bar{\gamma}, \stackrel{+}{\gamma}$-plane, the set of pairs $\bar{\gamma}, \stackrel{+}{\gamma}$ for which $\bar{\gamma}$ is in phase $1, \stackrel{+}{\gamma}$ is in phase 3 , and the right side of (3.8) is nonnegative is represented by the shaded region $\Gamma$ shown in Fig. 2. At any point on the boundary segment $B C$, the numerator in (3.8) vanishes, so that $\dot{s}=0$. The corresponding phase boundary is thus instantaneously stationary. In the special case of time-independent fields, points $(\bar{\gamma}, \stackrel{+}{\gamma})$ on $B C$ correspond to 


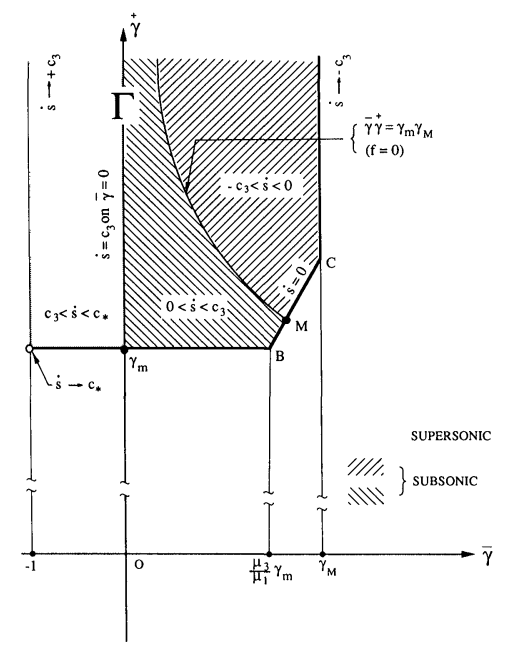

FIG. 2. The region $\Gamma$ in the $\bar{\gamma}, \dot{\gamma}$-plane.

equilibrium phase mixtures. If $\sigma_{0}$ denotes the equal-area stress in Fig. 1, the special equilibrium mixture for which $\bar{\gamma}=\sigma_{0} / \mu_{1}, \stackrel{+}{\gamma}=\sigma_{0} / \mu_{3}$ is called the Maxwell state; it corresponds to point $M$ in Fig. 2. The Maxwell state represents stable equilibrium; other phase-1, phase-3 equilibria are merely metastable.

The driving traction $f$ acting on a phase-1, phase- 3 interface can be found from (3.4) and the explicit form of $\hat{\sigma}(\gamma)$ for the trilinear material: one finds that

$$
f=\hat{f}(\bar{\gamma}, \stackrel{+}{\gamma})=\frac{1}{2}\left(\mu_{1}-\mu_{3}\right)\left(\gamma_{M} \gamma_{m}-\bar{\gamma} \dot{\gamma}\right)
$$

It follows that the driving traction vanishes on the hyperbola $\bar{\gamma} \dot{\gamma}=\gamma_{M} \gamma_{m}$. In view of (3.3), this means that points in $\Gamma$ that lie on this hyperbola correspond to values of $\bar{\gamma}$ and $\stackrel{+}{\gamma}$ for which the associated phase boundary $x=s(t)$ propagates without dissipation. Note that the Maxwell point $M$ lies on the hyperbola. At points off the hyperbola, $f \neq 0$, so that the entropy admissiblity requirement (3.5) determines the sign of $\dot{s}$ (see Fig. 2). For points on the hyperbola $f=0$, the sign of $\dot{s}$ is not determined by (3.5), and propagation in either direction is possible.

With the help of (3.8) and (3.5), we can show (as in [1]) that $\dot{s}$ necessarily lies in the range

$$
-c_{3}<\dot{s}<c_{*},
$$

where

$$
c_{*}=\left(\frac{c_{1}^{2}+\gamma_{m} c_{3}^{2}}{1+\gamma_{m}}\right)^{1 / 2}, \quad c_{3}<c_{*}<c_{1} .
$$

The propagation speed $\dot{s}$ is said to be subsonic if $|\dot{s}|<c_{3}$. From (3.8), it follows that this occurs only for those points in the region $\Gamma$ of Fig. 2 for which $\bar{\gamma}>0$. For supersonic motion of the phase boundary, $\bar{\gamma}$ must be negative, so that part of the bar is in compression. Furthermore, the driving traction is positive, so that the entropy admissibility requirement forces the direction of propagation of the phase boundary to be such that the portion of the bar in phase 1 grows at the expense of phase 3 . Thus for a supersonically propagating phase boundary, the "parent" phase-the phase into which the interface moves-is always phase 3. 
We now use (3.8) and (3.9) to map the region $\Gamma$ of the $\bar{\gamma}, \stackrel{+}{\gamma}$-plane into the $\dot{s}$, $f$-plane. Each point $(\bar{\gamma}, \stackrel{+}{\gamma})$ that does not lie on $B C$ (Fig. 2) is carried to two points $(\dot{s}, f)$ and $(-\dot{s}, f)$ in the $\dot{s}, f$-plane; if $f \neq 0$, only one of these satisfies the entropy admissibility requirement (3.5). If $f=0$, the point $(\bar{\gamma}, \stackrel{+}{\gamma})$ lies on the hyperbola; such a point maps to a pair of admissible points $( \pm \dot{s}, 0)$ in the $\dot{s}, f$-plane, with $0 \leqq \dot{s} \leqq c_{3}$. Each point on $B C$ maps to a single admissible point $(0, f)$ on the $f$-axis. All points in $\Gamma$ that lie on $\bar{\gamma}=0$ map to the same pair of points $\left( \pm c_{3}, f_{0}\right)$, where the constant $f_{0}$ is given by $f_{0}=\left(\mu_{1}-\mu_{3}\right) \gamma_{m} \gamma_{M} / 2>0$. Figure 3 shows the admissible image of $\Gamma$ in the $\dot{s}$, $f$-plane.

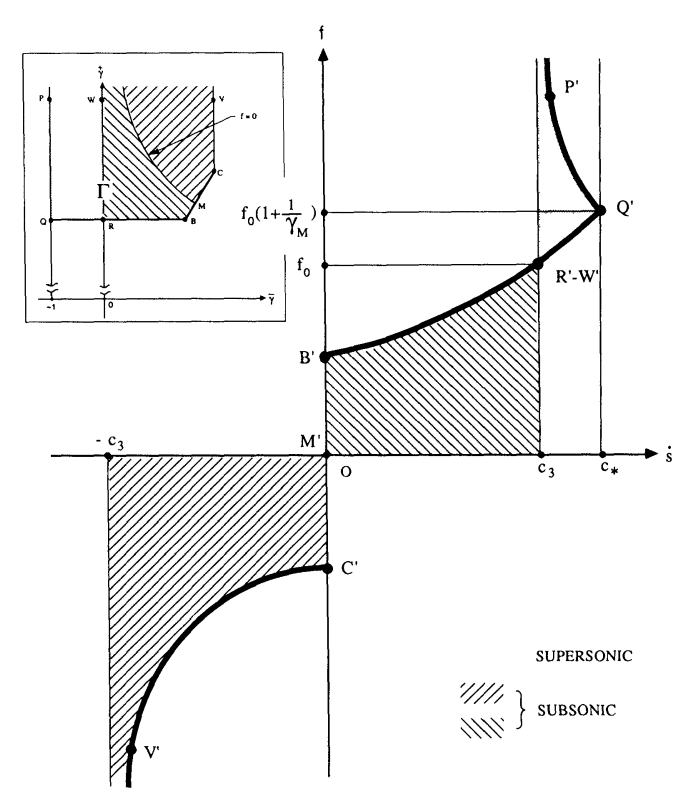

FIG. 3. Admissible image of $\Gamma$ (inset) in $\dot{s}, f$-plane.

The physical basis for the elucidation of phase transitions in solids involves both a nucleation criterion governing the initiation of the transition and a kinetic relation controlling the rate at which it proceeds (see [2], [17], [18]). We have discussed simple continuum-mechanical implementations of these notions in [1], [3], and [16]. In particular, it is shown in [1] that, as long as all phase boundaries propagate subsonically, a nucleation criterion and a kinetic relation can be imposed in the Riemann problem, and that they single out unique admissible solutions in that context. In the present discussion, we are concerned only with the propagation of an existing phase boundary and not with its emergence; the nucleation criterion is therefore not relevant here. As for the kinetic relation, we proceed as in [1] and assume that, if the phase boundary velocity $\dot{s}$ is subsonic, there is a function $\varphi$ determined by the material that relates the driving traction $f$ to $\dot{s}$, the latter being a measure of the rate at which the phase transition takes place. Thus we take

$$
f=\varphi(\dot{s}), \quad-c_{3}<\dot{s}<c_{3} .
$$

Because of the entropy admissibility requirement (3.5), $\varphi$ must satisfy

$$
\varphi(\dot{s}) \dot{s} \geqq 0, \quad-c_{3}<\dot{s}<c_{3} .
$$


It may be observed that if $\varphi(\dot{s})$ is continuous at $\dot{s}=0,(3.13)$ requires that $\varphi(0)=0$. It is required that the curve represented by (3.12) lie in the hatched region of the $\dot{s}, f$-plane shown in Fig. 3. The pre-image of this curve in the $\bar{\gamma}, \stackrel{+}{\gamma}$-plane under the mapping (3.8), (3.9), (3.5) thus comprises the locus of all strain-pairs $\bar{\gamma}, \stackrel{+}{\gamma}$ at a phase-1, phase-3 interface that are consistent with the kinetic relation (3.12).

The choice $\varphi(\dot{s}) \equiv 0$ for the kinetic response function corresponds to dissipationfree motions of the bar. For nearly dissipation-free motions, the choice

$$
\varphi(\dot{s})=\kappa \dot{s}, \quad-c_{3}<\dot{s}<c_{3},
$$

with $\kappa$ a sufficiently small nonnegative constant, is natural. Kinetic relations other than (3.14) can, of course, be considered. The choice

$$
\varphi(\dot{s})=K \sinh ^{-1}(\dot{s} / k),
$$

where $K$ and $k$ are constants, can be motivated by arguments of the kind used in thermal activation theory; for a sketch of the latter theory, see Chapter 1 of [17]. Other special choices of $\varphi(\dot{s})$ are discussed in [1] and [3]. Kinetic relations not of the form (3.12) may be of interest. One that is suggested by a dislocation-based theory put forward in [18] for the modeling of fast martensitic phase transitions is

$$
f=f_{*}+m \ddot{s}+\kappa \dot{s},
$$

with $f_{*}, m$, and $\kappa$ constants.

The kinetic relations discussed above represent local requirements, as indeed they must if they are to be regarded as part of the constitutive description of the material. Moreover, the notion of a scalar driving traction, and consequently that of a kinetic relation, can be carried over to more general constitutive settings in three dimensions with thermal effects accounted for as well; for further discussion, see [16].

It was shown in [1] that the Riemann problem based on the theory of elastic bars described above is not overdetermined when the kinetic relation (3.12) is imposed at subsonically propagating phase boundaries.

4. The augmented theory: construction of solutions. To pursue the approach based on the augmented theory, we replace the constitutive law (2.6) by (2.8), and we replace the moving discontinuity with strains $\stackrel{ \pm}{\gamma}$ and particle velocities $\stackrel{ \pm}{v}$ in the elastic bar by a traveling wave in the augmented theory. In the traveling wave,

$$
\gamma=\gamma(\xi), \quad v=v(\xi), \quad \xi=x-\dot{s} t,
$$

where $\dot{s}$ is a constant, and

$$
\gamma(-\infty)=\bar{\gamma}, \quad v(-\infty)=\bar{v}, \quad \gamma(+\infty)=\stackrel{+}{\gamma}, \quad v(+\infty)=\stackrel{+}{v}
$$

corresponding to a smooth connection of the states $\bar{\gamma}, \bar{v}$ and $\dot{\gamma}, \stackrel{+}{v}$. As before, $\bar{\gamma}$ is to be in phase $1, \stackrel{+}{\gamma}$ in phase 3 . In conformity with the smoothness assumptions made in $\S 2$, we suppose that $\gamma(\xi)$ and $v(\xi)$ are continuous and continuously differentiable on $(-\infty, \infty)$, and that they have piecewise continuous second and third derivatives there. In view of (2.4) and (2.8), $\gamma^{\prime \prime}(\xi)$ must, in fact, be continuous for all $\xi$, provided $\lambda \neq 0$.

From (2.2), (2.3), and (2.8), traveling waves of the form (4.1) must satisfy

$$
\begin{gathered}
\rho \lambda \gamma^{\prime \prime \prime}+\rho \nu \dot{s} \gamma^{\prime \prime}-\hat{\sigma}^{\prime}(\gamma) \gamma^{\prime}-\rho \dot{s} v^{\prime}=0, \\
v^{\prime}+\dot{s} \gamma^{\prime}=0,
\end{gathered}
$$

at all points where $\gamma^{\prime \prime \prime}(\xi)$ exists. We seek solutions $\gamma, v$ of $(4.3),(4.4)$ that satisfy the conditions (4.2) at $\pm \infty$. 
Before constructing an explicit solution to this boundary value problem, we record some useful observations. Assuming the existence of a solution, we first integrate (4.3), (4.4) to obtain

$$
\begin{gathered}
\lambda \gamma^{\prime \prime}+\nu \dot{s} \gamma^{\prime}-\frac{1}{\rho} \hat{\sigma}(\gamma)-\dot{s} v=A, \\
v+\dot{s} \gamma=B,
\end{gathered}
$$

where $A$ and $B$ are constants. From the boundary conditions (4.2), it follows that $A$ and $B$ satisfy

$$
A=-\frac{1}{\rho} \hat{\sigma}(\stackrel{+}{\gamma})-\dot{s} \hat{v}=-\frac{1}{\rho} \hat{\sigma}(\bar{\gamma})-\dot{s} \bar{v}, \quad B=\stackrel{+}{v}+\dot{s} \hat{\gamma}=\bar{v}+\dot{s} \bar{\gamma}
$$

But (4.7) immediately imply (3.6) and (3.7), which are therefore necessary conditions on $\dot{s}$ and the given data for the existence of a solution to the problem (4.2)-(4.4).

Elimination of $v$ between (4.3) and (4.4) yields a differential equation for $\gamma$ :

$$
\lambda \gamma^{\prime \prime}+\nu \dot{s} \gamma^{\prime}-\frac{1}{\rho} \hat{\sigma}(\gamma)+\dot{s}^{2} \gamma=C \quad \text { on }(-\infty, \infty)
$$

where the constant $C$ is given by

$$
C=A+\dot{s} B=-\frac{+}{\rho}+\dot{s}^{2} \dot{\gamma}=-\frac{\bar{\sigma}}{\rho}+\dot{s}^{2} \bar{\gamma},
$$

and we have written $\stackrel{ \pm}{\sigma} \equiv \hat{\sigma}\left(\frac{ \pm}{\gamma}\right)$. The boundary conditions associated with $(4.8)$ are

$$
\gamma(-\infty)=\bar{\gamma}, \quad \gamma(+\infty)=\stackrel{+}{\gamma},
$$

with $\bar{\gamma}$ in phase $1, \stackrel{+}{\gamma}$ in phase 3 . We speak of (4.8)-(4.10) as the traveling wave problem. Once $\gamma(\xi)$ has been found, $v(\xi)$ is determined from (4.6).

After multiplying the differential equation (4.8) by $\gamma^{\prime}(\xi)$ and integrating with respect to $\xi$ from $-\infty$ to $+\infty$, we find with the help of $(3.6),(4.9)$ that a solution $\gamma(\xi)$, if it exists, must satisfy

$$
\hat{f}(\bar{\gamma}, \stackrel{+}{\gamma})=\rho \nu \dot{s} \int_{-\infty}^{\infty}\left[\gamma^{\prime}(\xi)\right]^{2} d \xi
$$

where $\hat{f}$ is the function defined in (3.4). From (4.11) and the fact that $\nu \geqq 0$, it follows that, if the traveling wave problem has a solution, then $\dot{s}$ and the data $\bar{\gamma}, \stackrel{+}{\gamma}$ necessarily satisfy

$$
\hat{f}(\bar{\gamma}, \stackrel{+}{\gamma}) \dot{s} \geqq 0
$$

cf. the entropy admissibility condition (3.5) of the elastic theory. Of the two necessary conditions (3.6) and (4.12), the first determines the speed of propagation $|\dot{s}|$ of the traveling wave. The sign of $\dot{s}$, and hence the direction of propagation, is then determined by (4.12) with the help of (3.4), provided the given strains $\stackrel{ \pm}{\gamma}$ are such that $\hat{f}(\bar{\gamma}, \stackrel{+}{\gamma}) \neq 0$. As in the derivation of (3.10), from (3.6) and (4.12) we find that $\dot{s}$ lies in the interval $\left(-c_{3}, c_{*}\right)$.

We seek a solution of the traveling wave problem (4.8)-(4.10) for which $\gamma(\xi)$ is in phase 1 for $-\infty<\xi \leqq 0$, in phase 2 for $0<\xi<b$, and in phase 3 for $b \leqq \xi<\infty$, for some suitable $b$ to be determined. In view of our smoothness assumptions, the following conditions must hold at the interfaces between the various phases:

$$
\begin{array}{lll}
\gamma(0-)=\gamma_{M}, & \gamma(0+)=\gamma_{M}, & \gamma^{\prime}(0-)=\gamma^{\prime}(0+), \\
\gamma(b-)=\gamma_{m}, & \gamma(b+)=\gamma_{m}, & \gamma^{\prime}(b-)=\gamma^{\prime}(b+) .
\end{array}
$$


If (4.13), (4.14), and the differential equation (4.8) all hold, then $\gamma^{\prime \prime}(\xi)$ is automatically continuous at $\xi=0$ and $\xi=b$, provided $\lambda \neq 0$.

To construct such a solution, we first consider three subsidiary problems for the restrictions of $\gamma$ to the separate intervals $(-\infty, 0],[0, b]$, and $[b, \infty)$. The first of these is a boundary-value problem, while the second and third are initial-value problems; the problems are to be solved in order.

PROBLEM 1.

$$
\begin{gathered}
\lambda \gamma^{\prime \prime}+\nu \dot{s} \gamma^{\prime}-\left(c_{1}^{2}-\dot{s}^{2}\right) \gamma=C \text { on }(-\infty, 0], \\
\gamma(-\infty)=\bar{\gamma}, \quad \gamma(0-)=\gamma_{M} .
\end{gathered}
$$

Problem 2.

$$
\begin{gathered}
\lambda \gamma^{\prime \prime}+\nu \dot{s} \gamma^{\prime}+\left(c_{2}^{2}+\dot{s}^{2}\right) \gamma=C+\sigma_{2} / \rho \quad \text { on }[0, b], \\
\gamma(0+)=\gamma_{M}, \quad \gamma^{\prime}(0+)=\gamma^{\prime}(0-) .
\end{gathered}
$$

Problem 3.

$$
\begin{gathered}
\lambda \gamma^{\prime \prime}+\nu \dot{s} \gamma^{\prime}-\left(c_{3}^{2}-\dot{s}^{2}\right) \gamma=C \quad \text { on }[b, \infty), \\
\gamma(b+)=\gamma_{m}, \quad \gamma^{\prime}(b+)=\gamma^{\prime}(b-) .
\end{gathered}
$$

The function $\gamma$ on $(-\infty, \infty)$ determined by solving these problems will satisfy the differential equation (4.8), the first of the boundary conditions (4.10), all of the interface conditions (4.13) at $\xi=0$, and all but the first of the interface conditions (4.14) at $\xi=b$. Thus after solving Problems 1-3, we will still need to enforce the boundary condition

$$
\gamma(+\infty)=\stackrel{+}{\gamma}
$$

the interface condition

$$
\gamma(b-)=\gamma_{m}
$$

and the requirements

$$
\begin{array}{ll}
-1<\gamma(\xi) \leqq \gamma_{M} & \text { for }-\infty<\xi \leqq 0, \\
\gamma_{M}<\gamma(\xi)<\gamma_{m} & \text { for } 0<\xi<b, \\
\gamma(\xi) \geqq \gamma_{m} & \text { for } \xi \geqq b .
\end{array}
$$

The inequalities (4.23) ensure that the strain belongs to the appropriate phase in each interval.

After using the rightmost representation of $C$ in (4.9), one finds that the solution of Problem 1 is given by

$$
\gamma(\xi)=\bar{\gamma}+\left(\gamma_{M}-\bar{\gamma}\right) e^{p_{1} \xi}, \quad-\infty<\xi \leqq 0,
$$

where the positive number $p_{1}$ is defined by

$$
p_{1}=-\frac{\nu}{2 \lambda}(\dot{s}-P)>0, \quad P=P(\dot{s})=\left[\dot{s}^{2}+\omega^{2}\left(c_{1}^{2}-\dot{s}^{2}\right)\right]^{1 / 2}>0,
$$

and $\omega$ is defined in (2.9).

We turn next to Problem 2. The general solution of (4.17) is

$$
\gamma(\xi)=\gamma_{0}+D_{1} e^{q_{1} \xi}+D_{2} e^{q_{2} \xi}, \quad 0 \leqq \xi \leqq b,
$$


where $D_{1}$ and $D_{2}$ are arbitrary constants, and the constant $\gamma_{0}$ is defined by

$$
\gamma_{0}=\left(C+\sigma_{2} / \rho\right) /\left(c_{2}^{2}+\dot{s}^{2}\right)
$$

In (4.26), the (possibly complex) constants $q_{1}$ and $q_{2}$ in the exponentials are given by

$$
q_{1}=-\frac{\nu}{2 \lambda}(\dot{s}-Q), \quad q_{2}=-\frac{\nu}{2 \lambda}(\dot{s}+Q)
$$

where we have set

$$
Q=Q(\dot{s})= \begin{cases}{\left[\dot{s}^{2}-\omega^{2}\left(c_{2}^{2}+\dot{s}^{2}\right)\right]^{1 / 2}} & \text { if } \dot{s}^{2}-\omega^{2}\left(c_{2}^{2}+\dot{s}^{2}\right) \geqq 0 \\ i\left[\omega^{2}\left(c_{2}^{2}+\dot{s}^{2}\right)-\dot{s}^{2}\right]^{1 / 2} & \text { if } \dot{s}^{2}-\omega^{2}\left(c_{2}^{2}+\dot{s}^{2}\right) \leqq 0\end{cases}
$$

We now choose the constants $D_{1}$ and $D_{2}$ so that the initial conditions (4.18) for Problem 2 are satisfied. This leads to

$$
D_{1}=-\frac{\lambda}{\nu Q}\left(\gamma_{M}-\gamma_{0}\right) \frac{q_{2}}{p_{2}}\left(p_{2}-q_{1}\right), \quad D_{2}=+\frac{\lambda}{\nu Q}\left(\gamma_{M}-\gamma_{0}\right) \frac{q_{1}}{p_{2}}\left(p_{2}-q_{2}\right)
$$

here $p_{2}$ is given by

$$
p_{2}=-\frac{\nu}{2 \lambda}(\dot{s}+P)<0
$$

and $P$ is defined in (4.25). It has been assumed above that $Q \neq 0$, so that $q_{1} \neq q_{2}$; while the case $Q=0$ can be readily treated, we exclude it for simplicity. Note that although $q_{1}$ and $q_{2}$ may be either real or complex, $\gamma$ as given on $[0, \mathrm{~b}](4.26)-(4.31)$ is always real.

Finally, we consider Problem 3. Its solution is given by

$$
\gamma(\xi)=\stackrel{+}{\gamma}+\frac{\gamma_{m}-\stackrel{+}{\gamma}}{r_{1}-r_{2}}\left[-r_{2} e^{r_{1}(\xi-b)}+r_{1} e^{r_{2}(\xi-b)}\right]+\frac{\gamma^{\prime}(b-)}{r_{1}-r_{2}}\left[e^{r_{1}(\xi-b)}-e^{r_{2}(\xi-b)}\right],
$$

$\xi \geqq b$,

where $r_{1}$ and $r_{2}$ are defined by

$$
r_{1}=-\frac{\nu}{2 \lambda}(\dot{s}-R), \quad r_{2}=-\frac{\nu}{2 \lambda}(\dot{s}+R)
$$

and we have set

$$
R=R(\dot{s})= \begin{cases}{\left[\dot{s}^{2}+\omega^{2}\left(c_{3}^{2}-\dot{s}^{2}\right)\right]^{1 / 2}} & \text { if } \dot{s}^{2}+\omega^{2}\left(c_{3}^{2}-\dot{s}^{2}\right) \geqq 0 \\ i\left[\omega^{2}\left(\dot{s}^{2}-c_{3}^{2}\right)-\dot{s}^{2}\right]^{1 / 2} & \text { if } \dot{s}^{2}+\omega^{2}\left(c_{3}^{2}-\dot{s}^{2}\right) \leqq 0\end{cases}
$$

In (4.32), $\gamma^{\prime}(b-)$ is found from (4.26):

$$
\gamma^{\prime}(b-)=D_{1} q_{1} e^{q_{1} b}+D_{2} q_{2} e^{q_{2} b},
$$

with $D_{1}$ and $D_{2}$ given by (4.30). For simplicity, we have excluded the case $R=0$, corresponding to $r_{1}=r_{2}$. Again, we note that, although $r_{1}$ and $r_{2}$ may be either real or complex, $\gamma(\xi)$ as given on $[b, \infty)$ by $(4.32)-(4.35),(4.28)-(4.30)$ is always real.

To complete the construction of a solution $\gamma(\xi)$ to the traveling wave problem from the solutions of Problems 1-3 it is necessary to address the remaining requirements (4.21)-(4.23). By (4.26), we may write (4.22) as

$$
D_{1} e^{q_{1} b}+D_{2} e^{q_{2} b}=\gamma_{m}-\gamma_{0},
$$

where $D_{1}$ and $D_{2}$ are given by (4.30), $q_{1}$ and $q_{2}$ by (4.28), and $\gamma_{0}$ by (4.27). Equation (4.36) is a condition for the determination of the length $b$ of the phase- 2 interval; we postpone the discussion of the solvability of (4.36). 
Of the requirements (4.23), the first pertains to the interval $(-\infty, 0]$; by $(4.24)$, this condition is automatically satisfied. We will address those conditions in (4.23) that pertain to $(0, b)$ and $[b, \infty)$ after $b$ has been determined.

Finally, we note from (4.32) that, to discuss the boundary condition (4.21) at $\xi=+\infty$, we must know the sign of the real parts of $r_{1}$ and $r_{2}$. These signs depend critically on whether the traveling wave is moving subsonically $\left(|\dot{s}|<c_{3}\right)$ or supersonically $\left(\dot{s}>c_{3}\right)$. In continuing the analysis, we treat these two cases separately.

5. The augmented theory: subsonic case. Suppose first that the given strains $\stackrel{ \pm}{\gamma}$ at infinity are such that the propagation speed $\dot{s}$ delivered by (3.6) is subsonic: $\dot{s}^{2}<c_{3}^{2}$. Then by (4.33), (4.34), $r_{1}$ and $r_{2}$ are both real, and $r_{1}>0, r_{2}<0$. From (4.32), it then follows that $\gamma(\xi)$ satisfies the boundary condition $\gamma(+\infty)=\stackrel{+}{\gamma}$ if and only if the coefficient of $\exp \left[r_{1}(\xi-b)\right]$ in (4.32) vanishes. Making use of (4.35), we may write this condition as

$$
D_{1} q_{1} e^{q_{1} b}+D_{2} q_{2} e^{q_{2} b}=r_{2}\left(\gamma_{m}-\stackrel{+}{\gamma}\right) .
$$

Observe that both (4.36) and (5.1) must hold with $D_{1}$ and $D_{2}$ given by (4.30). Thus if one of these, say (4.36), serves to determine $b$, the remaining one-(5.1)_provides $a$ further necessary restriction on the given data $\stackrel{ \pm}{\gamma}$.

With (5.1) in force, only the exponentials involving $r_{2}$ remain in (4.32), so that $\gamma(\xi)$ increases monotonically from $\gamma_{m}$ at $\xi=b$ to $\stackrel{+}{\gamma}$ at $\xi=+\infty$, thereby satisfying $(4.23)_{3}$ and thus assuring that $\gamma(\xi)$ is indeed in phase 3 for all $\xi \geqq b$.

After some algebra that makes use of (2.7), (4.9), (4.25), and (4.27)-(4.33), we find that (4.36) and (5.1) are equivalent to the following pair of equations:

$$
\begin{gathered}
e^{Q \nu b / \lambda}=\left(\frac{Q-P}{Q+P}\right)\left(\frac{Q-R}{Q+R}\right), \\
e^{-\nu \dot{s} b / \lambda}=\left(\frac{\dot{s}+P}{\dot{s}-R}\right)^{2} \frac{\gamma_{M}}{\gamma_{m}}\left(\frac{\gamma_{m}-\gamma_{0}}{\gamma_{M}-\gamma_{0}}\right)^{2},
\end{gathered}
$$

where $Q, P, R$, and $\gamma_{0}$ are given by (4.29), (4.25) $2,(4.34)_{1}$, and (4.27), respectively. We regard (5.2) as an equation for $b$, so that (5.3) becomes the restriction on the data alluded to above.

To analyze (5.2), we must distinguish between real and complex $Q$. It follows from (4.29) and the fact that $|\dot{s}|<c_{3}$ that $Q$ is complex and different from zero if and only if

$$
|\dot{s}|<c_{* *}
$$

where

$$
c_{* *}=c_{* *}(\omega) \equiv \begin{cases}{\left[\omega^{2} /\left(1-\omega^{2}\right)\right]^{1 / 2} c_{2} \leqq c_{3}} & \text { if } 0<\omega^{2} \leqq c_{3}^{2} /\left(c_{2}^{2}+c_{3}^{2}\right), \\ c_{3} & \text { if } \omega^{2} \geqq c_{3}^{2} /\left(c_{2}^{2}+c_{3}^{2}\right) .\end{cases}
$$

Figure 4 shows the regions in the $\bar{\gamma}, \stackrel{+}{\gamma}$-plane that correspond to real and to complex $Q$.

We now consider (5.2). Suppose first that $Q$ is real, and therefore positive. In this case, the left side of (5.2) increases with $b$ from the value 1 at $b=0$ to $+\infty$ at $b=+\infty$, but the right side of (5.2) is less than unity. It follows that (5.2) cannot hold for any $b>0$ when $Q$ is real. Thus any point in either of the unshaded areas in Fig. 4 corresponds to a pair of strains $\bar{\gamma}, \stackrel{+}{\gamma}$ that cannot be connected by a subsonic traveling wave of the assumed form. 


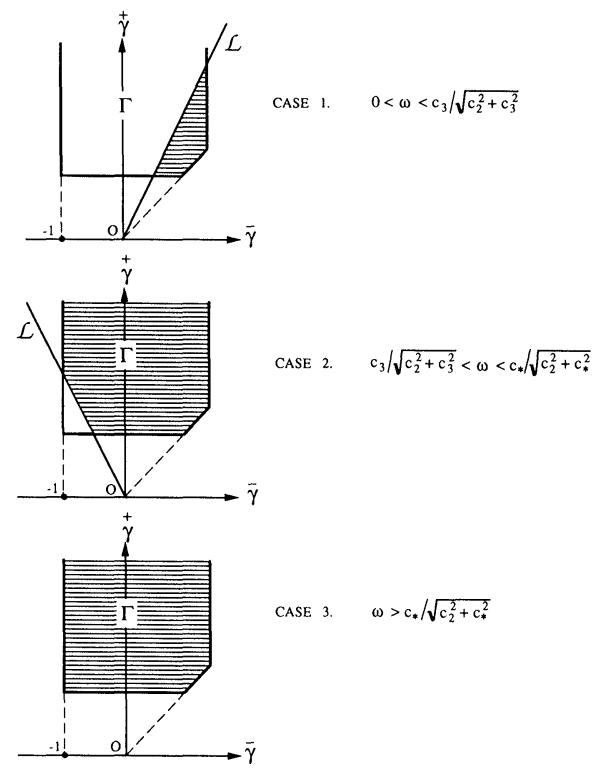

FIG. 4. Subregions of $\Gamma$ corresponding to real and complex $Q . \mathscr{L}: \bar{\gamma}=\Delta \bar{\gamma}, \Delta=\left[\left(1-\omega^{2}\right) c_{1}^{2}-\omega^{2} c_{2}^{2}\right] /$ $\left[\left(1-\omega^{2}\right) c_{3}^{2}-\omega^{2} c_{2}^{2}\right] . Q$ is complex in hatched regions.

By (4.29), if $Q$ is complex, it is pure imaginary, and both sides of (5.2) represent complex numbers on the unit circle. It follows that (5.2) has infinitely many roots. Let $\theta_{P}$ and $\theta_{R}$ stand for the respective arguments of the complex numbers $P+Q=P+i|Q|$ and $R+Q=R+i|Q|$. Since $P, R$, and $|Q|$ are positive, $\theta_{P}$ and $\theta_{R}$ may both be taken in $(0, \pi / 2)$. The positive roots of $(5.2)$ are given by

$$
b=b_{n}=\frac{\lambda}{\nu|Q|}\left[2(n+1) \pi-2\left(\theta_{P}+\theta_{R}\right)\right], \quad n=0,1,2, \cdots .
$$

It can be shown that, of all the roots $b_{n}$ listed above, only $b=b_{0}$ furnishes an interval $(0, b)$ on which $\gamma(\xi)$ as given by $(4.26)-(4.31)$ is everywhere in phase 2 ; indeed, when $b=b_{0}, \gamma(\xi)$ increases monotonically on $[0, b]$. Thus (5.2) determines exactly one acceptable value of $b$.

Having found $b$, we turn to (5.3). To interpret this restriction on the data, we first note that $(5.3)$ can be solved for $\gamma_{0}$ in terms of $\dot{s}$ :

$$
\gamma_{0}=\gamma_{M}+\frac{\gamma_{m}-\gamma_{M}}{1+\left(\gamma_{m} / \gamma_{M}\right)^{1 / 2}((R-\dot{s}) /(P+\dot{s})) e^{-\nu \dot{s} b / 2 \lambda}}
$$

From (2.7), (4.9), and (4.27), we can show that

$$
\bar{\gamma}=\gamma_{M}+\frac{c_{2}^{2}+\dot{s}^{2}}{c_{1}^{2}-\dot{s}^{2}}\left(\gamma_{M}-\gamma_{0}\right), \quad \stackrel{+}{\gamma}=\gamma_{m}+\frac{c_{2}^{2}+\dot{s}^{2}}{c_{3}^{2}-\dot{s}^{2}}\left(\gamma_{m}-\gamma_{0}\right) .
$$

Using (5.7) in (5.8) and eliminating $c_{2}$ with the help of (2.7) ultimately yields

$$
\bar{\gamma}=\frac{G(\dot{s})}{c_{1}^{2}-\dot{s}^{2}}, \quad \stackrel{+}{\gamma}=\frac{G(\dot{s})}{c_{3}^{2}-\dot{s}^{2}}, \quad-c_{* *}<\dot{s}<c_{* *},
$$

where $G(\dot{s})$ is given by

$$
G(\dot{s})=\frac{H(\dot{s})}{1+H(\dot{s})}\left(c_{1}^{2}-\dot{s}^{2}\right) \gamma_{M}+\frac{1}{1+H(\dot{s})}\left(c_{3}^{2}-\dot{s}^{2}\right) \gamma_{m}, \quad-c_{* *}<\dot{s}<c_{* *},
$$


and

$$
H(\dot{s})=\left(\frac{\gamma_{m}}{\gamma_{M}}\right)^{1 / 2} \frac{R(\dot{s})-\dot{s}}{P(\dot{s})+\dot{s}} e^{-\nu \dot{s} b / 2 \lambda}>0, \quad-c_{* *}<\dot{s}<c_{* *} .
$$

In (5.11), $R$ and $P$ are given by (4.34) $)_{1}$ and (4.25) $)_{2}$, respectively, and $b$ is understood to be $b_{0}$ (see (5.6)). Equation (5.9) provides a new representation for the original restriction (5.3) on the given data. Since by $(4.25)_{2},(4.29)_{2}$, and $(4.34)_{1}, P, Q$, and $R$ depend on the constants $\lambda$ and $\nu$ of the augmented theory only through the parameter $\omega$ given by (2.9), it follows from (5.6), (5.11), and (5.10) that $\nu b / \lambda, H$, and $G$ have this property as well.

In summary, we have shown that if there is a subsonic traveling wave of the assumed form connecting a phase- 1 strain $\bar{\gamma}$ at $\xi=-\infty$ to a phase- 3 strain $\stackrel{+}{\gamma}$ at $\xi=+\infty$, and if neither $Q$ nor $R$ vanishes, then $\gamma(\xi)$ must be given by (4.24), (4.26), and (4.32), $b$ must be given by (5.6) with $n=0$, and the given data $\bar{\gamma}, \stackrel{+}{\gamma}$ must satisfy the restriction (5.9).

Conversely, if the data fulfill (5.9) and if $b$ is given by (5.6) with $n=0$, then neither $Q$ nor $R$ vanishes, and $\gamma(\xi)$ as given by (4.24), (4.26), and (4.32) is a subsonic solution of the assumed form to the traveling wave problem (4.8)-(4.10).

6. The augmented theory: supersonic case. Next, let the given strains $\stackrel{ \pm}{\gamma}$ at infinity be such that $\dot{s}$ is supersonic: $c_{3}<\dot{s}<c_{*}$. In contrast to the subsonic case, $r_{1}$ and $r_{2}$ may now be either real or complex. However, according to (4.33) and (4.34), when $r_{1}$ and $r_{2}$ are real, both are negative, and when they are complex, both have negative real parts. It then follows that, in either case, the restriction of $\gamma(\xi)$ to $[b, \infty)$ as given by (4.32)-(4.34) satisfies the boundary condition $\gamma(+\infty)=\stackrel{+}{\gamma}$ without further restriction on the data $\stackrel{ \pm}{\gamma}$.

We now return to (4.36) to determine $b$ in the supersonic case. Detailed analysis of (4.36) shows that, when $q_{1}$ and $q_{2}$ are both real, (4.36) has exactly one positive root $b$, and this root is such that the strain $\gamma(\xi)$ associated with the restriction of $\gamma$ to $(0, b)$ is indeed always in phase 2 , as required. We can also show that, when $q_{1}$ and $q_{2}$ are complex, (4.36) has infinitely many positive roots $b$, but only the smallest of these leads to strains $\gamma(\xi)$ that belong to phase 2 for $0<\xi<b$. Thus whether $q_{1}$ and $q_{2}$ are real or complex, (4.36) determines exactly one acceptable value of $b$ in the supersonic case. Finally, it can be shown that, for this value of $b$, the strain $\gamma(\xi)$ associated with the restriction (4.32) of $\gamma$ to $[b, \infty)$ is in phase 3 for all $\xi \geqq b$ in those supersonic cases for which $r_{1}$ and $r_{2}$ in (4.33) are real. We have been unable to show that this conclusion persists in supersonic motions with complex $r$ 's, but numerical calculations suggest that it does. We will take this for granted in what follows.

In summary, if there is a supersonic traveling wave of the assumed form connecting a phase-1 strain $\bar{\gamma}$ at $\xi=-\infty$ to a phase- 3 strain $\stackrel{+}{\gamma}$ at $\xi=+\infty$, and if neither $Q$ nor $R$ vanishes, then a unique value of $b$ can be found, and $\gamma(\xi)$ must be given by (4.24), (4.26), and (4.32). Conversely, given $\bar{\gamma} \in(-1,0)$ and ${ }^{+} \gamma>\gamma_{m}$ (see Fig. 2) for which $Q$ and $R$ do not vanish, then for a suitable value of $b, \gamma(\xi)$ as given by (4.24), (4.26), and (4.32) represents a supersonic traveling wave without further restriction on the data $\stackrel{ \pm}{\gamma}$.

Finally, we note that the special case of the augmented theory for which $\lambda=0$, $\nu>0$ corresponds to a simple viscoelastic model of the bar. Although we do not treat this case in detail here, we remark that it leads to the conclusion that all traveling waves in the augmented theory are supersonic. A related observation was made by Slemrod [8] in motivating the inclusion of capillarity as well as viscosity when augmenting the theory of the van der Waals fluid. As noted in [4] and [18], slowly moving 
phase boundaries in solids have been observed in experiments; the special case $\lambda=0$ of the present augmented theory would therefore seem too special.

At the other extreme, the special case of the augmented theory in which $\nu=0$, $\lambda>0$ leads to purely dissipation-free behavior, as suggested by (4.11).

7. Permissible phase boundaries in the elastic bar. According to the criterion based on the augmented theory described here, permissible phase boundaries within the elastic theory are those for which the associated strains $\bar{\gamma}$ and $\stackrel{+}{\gamma}$ can be connected by a traveling wave in the augmented theory. For phase-1, phase-3 interfaces, pairs of strains $\bar{\gamma}, \stackrel{+}{\gamma}$ permitted by the jump conditions $(2.4),(2.5)$ in the elastic theory correspond to points in the region $\Gamma$ of Fig. 2. Among all points in $\Gamma$, those for which $-1<\bar{\gamma}<0$ corresponds to supersonic phase boundaries, and those with $0<\bar{\gamma}<\gamma_{M}$ are associated with subsonic phase boundaries.

As we have shown in the preceding section, every "supersonic pair" $\bar{\gamma}, \stackrel{+}{\gamma}$ (except possibly those for which either $\mathrm{R}$ or $\mathrm{Q}$ vanishes) can be connected by a traveling wave in the augmented theory. Thus all such supersonic pairs are "preferred" according to the present criterion.

In contrast, the results of $\S 5$ show that, among all points $(\bar{\gamma}, \stackrel{+}{\gamma})$ in $\Gamma$ that correspond to subsonically propagating phase boundaries, only those that satisfy the restriction (5.9) are such that $\bar{\gamma}$ can be connected to $\stackrel{+}{\gamma}$ by a traveling wave in the augmented theory. Interpreted geometrically, condition (5.9) is the parametric representation of a curve in the subsonic portion of $\Gamma$ that corresponds to the locus of pairs $\bar{\gamma}, \stackrel{+}{\gamma}$ that are preferred in the elastic theory according to the present criterion.

More significantly, the restriction (5.9) on preferred subsonic phase boundary strains is equivalent to a kinetic relation of precisely the form (3.12). By substituting for $\bar{\gamma}$ and $\stackrel{+}{\gamma}$ from (5.9) into the representation (3.9) for the driving traction $f$ at a phase-1, phase- 3 interface, we find indeed that

$$
f=\varphi(\dot{s}),
$$

where

$$
\varphi(\dot{s})=\frac{1}{2}\left(\mu_{1}-\mu_{3}\right)\left[\gamma_{M} \gamma_{m}-\frac{G^{2}(\dot{s})}{\left(c_{1}^{2}-\dot{s}^{2}\right)\left(c_{3}^{2}-\dot{s}^{2}\right)}\right], \quad-c_{* *}<\dot{s}<c_{* *} .
$$

In (7.2), $G(\dot{s})$ is given by $(5.10),(5.11)$.

It is appropriate to note here that the solution $\gamma(\xi)$ of the traveling wave problem in the augmented theory has a limit as the viscosity $\nu$ tends to zero, the parameter $\omega$ and the data $\bar{\gamma}, \dot{\gamma}$ being held fixed. Because $P, Q$, and $R$ depend only on $\omega$ and the data, it follows from (5.6) that the length $b$ of the phase-2 interval tends to zero in this limit. It is also readily shown that $\gamma(\xi)$ tends to $\bar{\gamma}$ for every $\xi<0$ and to $\dot{\gamma}$ for every $\xi>0$ as $\nu \rightarrow 0, \omega$ fixed. This zero-viscosity limit of $\gamma(\xi)$ satisfies all the differential equations and jump conditions of the elastic theory. Within that theory, it represents a propagating phase boundary moving with a velocity $\dot{s}$ that satisfies (3.8) and the entropy admissibility requirement (3.5). Moreover, because $G$ in (5.10) depends on $\nu$ and $\lambda$ only through $\omega$, the kinetic relation (7.2) remains unaffected by the limit process.

Figure 5 describes the special kinetic response function $\varphi$ of (7.2) by plotting $f / f_{0}$ versus $\dot{s} / c_{3}$ according to (7.1), (7.2); here $f_{0}=\left(\mu_{1}-\mu_{3}\right) \gamma_{M} \gamma_{m} / 2$. One finds that $f / f_{0}$ depends only on the three material parameters $\sigma_{M} / \sigma_{m}, \gamma_{M} / \gamma_{m}$, and $\omega$; the figure is plotted for fixed values of the first two of these and for various values of $\omega$. For small values of $\omega$ (corresponding, for example, to large viscosity), the graph strikes the top and bottom boundries of the permissible region in the $\dot{s}, f$-plane when $\dot{s}=c_{* *}$. This 


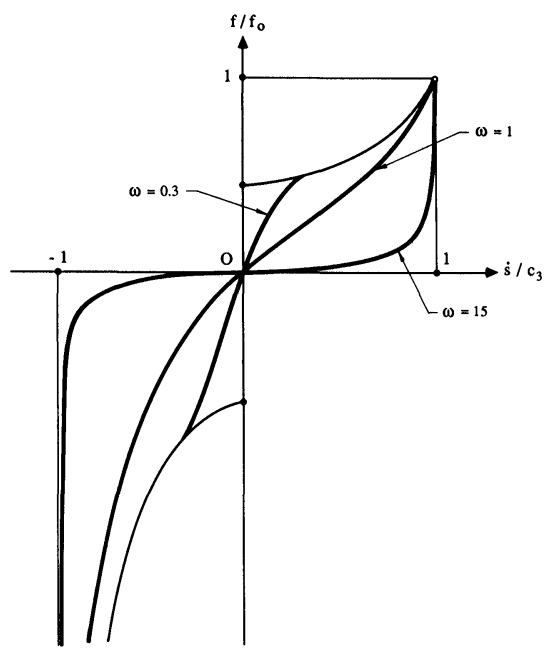

FIG. 5. The kinetic relation (7.1), (7.2) for $\sigma_{M} / \sigma_{m}=\frac{7}{4}, \gamma_{M} / \gamma_{m}=\frac{1}{2}$, and various values of $\omega$.

occurs when $\omega<c_{3} /\left(c_{2}^{2}+c_{3}^{2}\right)^{1 / 2}$ (see (5.5)). For the other values of $\omega$ considered, $f / f_{0}$ approaches $-\infty$ as $\dot{s} / c_{3}$ tends to -1 , but $f / f_{0}$ tends to unity as the latter ratio approaches +1 . For large values of $\omega$, or small viscosity, the kinetic relation corresponds to nearly dissipation-free motion of the phase boundary over most of the range of velocity $\dot{s}$. Note that, from the graphs, $\varphi(\dot{s})$ increases monotonically with $\dot{s}$ for the values of the parameters considered. The kinetic relations encompassed by the analysis in [1] were assumed to have this monotonicity property. It can be shown that, as indicated in Fig. 5 and as expected from (4.11) and (4.12), $\varphi(\dot{s})$ as given by (7.2) satisfies the requirement (3.13) imposed on every kinetic response function by the entropy admissibility condition (3.5).

The kinetics (7.1), (7.2) inherited from the particular augmented theory considered here correspond to a special case of those that have the form (3.12) and were considered in [1] and [3]. These in turn are by no means the most general kinetic relations conceivable. Moreover, augmentation is not the only way to specify the kinetics of a phase transition within a continuum model; thermal activation theory, for example, provides an entirely different approach that may be physically appropriate under certain circumstances. Even within the general framework of augmentation, alternatives suggest themselves: we might supplement the Newtonian viscous damping $\nu \gamma_{t}$ in (2.8) by adding a term proportional to $\gamma_{t}^{3}$; the resulting detailed kinetics would presumably differ from those described by (7.1) and (7.2). Indeed, it is unlikely that any scheme based on an augmented theory for modeling the kinetics of phase transitions in solids can lay claim to universality.

\section{REFERENCES}

[1] R. Abeyaratne And J. K. KnOwles, Kinetic relations and the propagation of phase boundaries in solids, Tech. Report No. 10, ONR contract N00014-87-K-0117, September 1989; Arch. Rational Mech. Anal., to appear.

[2] J. W. Christian, The Theory of Transformations in Metals and Alloys, Part I, Pergamon, Oxford, 1975.

[3] R. Abeyaratne And J. K. KNOWLes, On the dissipative response due to discontinuous strains in bars of unstable elastic material, Internat. J. Solids and Structures, 24 (1988), pp. 1021-1044.

[4] R. V. KRISHNAN AND L. C. Brown, Pseudo-elasticity and the strain-memory effect in an Ag-45 at. pct. Cd alloy, Metallurgical Trans., 4 (1973), pp. 423-429. 
[5] M. SHEARER, The Riemann problem for a class of conservation laws of mixed type, J. Differential Equations, 46 (1982), pp. 426-443.

[6] - Nonuniqueness of admissible solutions of Riemann initial value problems for a system of conservation laws of mixed type, Arch. Rational Mech. Anal., 93 (1986), pp. 45-59.

[7] — Dynamic phase transitions in a van der Waals gas, Quart. Appl. Math., 46 (1988), pp. 631-636.

[8] M. Slemrod, Admissibility criteria for propagating phase boundaries in a van der Waals fluid, Arch. Rational Mech. Anal., 81 (1983), pp. 301-315.

[9] - Dynamics of first order phase transitions, in Phase Transformations and Material Instabilities in Solids, M. E. Gurtin, ed., Academic Press, New York, 1984, pp. 163-203.

[10] _ A limiting "viscosity" approach to the Riemann problem for materials exhibiting change of phase, Arch. Rational Mech. Anal., 105 (1989), pp. 327-365.

[11] L. TRUSKINOvSKy, Equilibrium phase interfaces, Soviet Phys. Dokl., 27 (1982), pp. 551-553.

[12] - Structure of an isothermal phase discontinuity, Soviet Phys. Dokl., 30 (1985), pp. 945-948.

[13] Dynamics of non-equilibrium phase boundaries in a heat-conducting non-linearly elastic medium, J. Appl. Math. Mech. (PMM USSR), 51 (1987), pp. 777-784.

[14] D. J. KorteWEG, Sur la forme que prennent les équations du mouvement des fluides si l'on tient compte des forces capillaires causées par des variation de densité, Arch. Néerlandaises des Science Exactes et Naturelle, Ser. II, 6 (1901), pp. 1-24.

[15] J. E. DUNN AND J. SERRIN, On the thermomechanics of interstitial working, Arch. Rational Mech. Anal., 88 (1985), pp. 95-133.

[16] R. ABEyARATNE AND J. K. KNOWLES, On the driving traction acting on a surface of strain discontinuity in a continuum, J. Mech. Phys. Solids, 38 (1990), pp. 345-360.

[17] D. A. Porter ANd K. E. EAsterling, Phase Transformations in Metals and Alloys, van NostrandReinhold, New York, 1981.

[18] M. Grujicic, G. B. Olson, And W. S. OWen, Mobility of martensite interfaces, Metallurgical Trans. A, 16A (1985), pp. 1713-1722. 